\title{
Free-Energy-Based Analysis of Soft Transducers with Electroactive Polymers
}

\author{
Deok-Kee Choi* \\ Department of Mechanical Engieering, Dankook University, \\ 152, Jukjeon-ro, Suji-gu, Yongin-si, Gyeonggi-do, 448-701, Korea
}

(Received July 9, 2014; accepted December 8, 2014)

Key words: dielectric elastomer, transducers, electroactive polymers, nonlinear electrostatics, free energy, variational formulation

The application of electroactive polymers into soft transducers such as actuators, sensors or artificial muscles has become popular lately owing to the recent development of various numerical frameworks, which make it possible to analyze and simulate multiphysics problems with the help of computational methods. The energy formulation sets out to derive the free energy that contains information on the behavior of dielectric elastomer transducers under various electric fields or mechanical loadings, so that the energy-based formulation may meet the requirement of the principles of continuum mechanics and the laws of thermodynamics. The goal of the present study is, by applying the principle of virtual work to electromechanical problems, to set up a computational framework for which the linearization is carried out to be readily implemented into the finite element method to simulate the behavior of soft transducers under external stimuli.

\section{Introduction}

Electroactive polymers (EAPs) is a general term to designate dielectric elastomers (DEs) or other similar materials, which are sensitive to electric stimuli. Classes of EAP are categorized as electrostrictive, piezoelectric, and ferroelectric. ${ }^{(1-8)}$ In particular, DEs have some advantages over other conventional materials such as flexibility, scalability, high energy density, low weight, large deformation, and low cost. The application of electroactive smart materials in the realm of transducers has been rapidly growing lately, ${ }^{(9-11)}$ among which DEs have received much attention from various industrial sectors. A typical DE transducer is composed of a dielectric polymer sandwiched by compliant electrodes, as shown in Fig. 1. DEs are considered fundamentally as capacitor-like devices, which can change the capacitance when a voltage is applied, having the material squeezed in thickness and expand in area due to an electric field, that is, whose material properties can be changed rapidly upon the application of an electric field. DEs need a

*Corresponding author: e-mail: dkchoi@dku.edu 

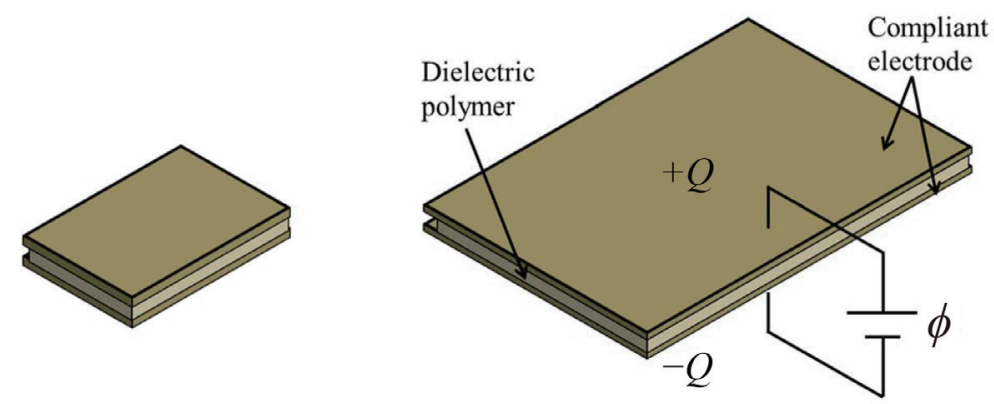

Fig. 1. (Color online) Schematic of a soft transducer made of dielectric elastomers, where $Q$ is the electric charge and $\phi$ is the electric potential.

high voltage to produce electric fields yet a low electrical power consumption, and do not need an electric field to keep an actuator at a given position.

Because of their low stiffness, DEs deform by a large amount, responding to external electric loads rapidly on the order of milliseconds. The application of deformation due to mechanical reactions of highly flexible polymers with respect to a change in an electric field is of great interest in the area of smart materials, particularly artificial muscles or robot fingers. For a robot finger to act like a flexible actuator, it is common to take mechanical deformation for input and an electric voltage change for output to be used as readings from a measuring device.

The range of topics related to DEs is enormous, including electrical response under high electric field, soft actuators and sensors, ferroelectric polymers, biomaterials, organic-based ferroelectric materials, energy-storing materials, energy-harvesting polymers, nonlinear electrical and mechanical properties, and electric phenomena in a nanoscale configuration.

Although DEs have many advantages over other conventional smart materials in both mechanical and electric performance characteristics, the complexity in the understanding of multi-physics phenomena has kept researchers from building proper numerical frameworks to grasp the whole picture of the subject at once, which may be mainly ascribed to the coupled nature of electroelastic phenomena. The behavior of a DE transducer under multi-field loadings is very interesting yet quite a complex phenomenon that it is not easily explicable by a simple theoretical framework. Macroscopic analysis of the subject calls for multifield theories accompanied by physics laws, balance equations, initial conditions, boundary conditions, and so forth. Furthermore, microscopic study of the behavior of DEs is extremely complicated if not impossible.

A theoretical framework that can achieve the combination of two different fields - the electric field and the mechanical field-has long been an interesting subject for people from both sides. ${ }^{(12-22)}$ It is not common to see that a researcher has sufficient knowledge of both mechanical and electric fields and he or she may be able to put two different disciplines into a unified framework. Even worse, the behavior of DEs is usually nonlinear, which demands numerical nonlinear analysis. 
The principle of virtual work (PVW) has been widely accepted and utilized to expand the area of such research. According to this principle, virtual work, which consists of electrostatics, and mechanical and thermodynamic energies, should meet a stationary condition. ${ }^{(23-26)}$ However, there has been much debate on how to distinguish the electric effect from a mechanical one owing to the presence of dielectrics, which has resulted in several different kinds of formulations to date. ${ }^{(27-33)}$ Many researchers have used the total stress that combines electromagnetic stress and mechanical stress into one.

According to nonlinear continuum mechanics, there are two kinds of descriptions of problems to be solved - the material description and the spatial descriptionfor example, the governing equations depending on the variables are expressed in a material configuration or spatial description. Either description gives the same result, so it is matter of preference to choose one description over the other. In general, a numerical formulation begins with the material configuration and is used to define constitutive relations through the push-forward operation; it is hence transformed into a spatial description. Besides electrostatic problems, some interesting topics such as micro-structural optimization, rate-dependent material models, and electromechanical instability of DE transducers have appeared in the literature.

Here, we limit the scope of the study to the large elastic deformation. To analyze complex material behavior problems occurring in DEs, there have been many studies regarding coupled phenomena such as the interaction between electric and mechanical fields.

Our goal in the present study is to develop a numerical framework in the material configuration, which is applicable in solving highly nonlinear and coupled electro-elastic problems. Fundamental equations in electrodynamics and nonlinear continuum mechanics are explained to set up a coupled multi-field analysis environment in $\S 2$. Based on thermodynamics equilibrium, to determine the deformation subjected to electric potential or vice versa, constitutive equations are obtained from free energy function. In $\S 3$, the variational equations derived through the principle of virtual work need to be linearized with respect to the displacement and the electric potential to reduce strong nonlinearity in the problems. Section 4 outlines the discretization of the equations for the finite element method and the linearization on the equations to be numerically solved. In $\S 5$, the procedure to build up a numerical framework is discussed. Finally, in the conclusion, the present work is summarized briefly.

\section{Electro-Continuum-Mechanics Equations}

\subsection{Kinematics}

Consider a body $B_{0}$ and a material particle $X \in B_{0}$ in the reference configuration. The particle in the current configuration $B$ for $\boldsymbol{x} \in B$ and the mapping function is given by $\boldsymbol{x}=\boldsymbol{\eta}(\boldsymbol{X}, t)$ at time $t$; the deformation gradient $\boldsymbol{F}$ is defined as

$$
\boldsymbol{F}=\frac{\partial \boldsymbol{\eta}}{\partial \boldsymbol{X}}=\nabla \boldsymbol{x}
$$


where $\nabla$ denotes the gradient with respect to the reference coordinate $X$. The surface of each boundary as shown in Fig. 2 is defined as $\partial B_{0}=\partial B_{0}^{u} \cup \partial B_{0}^{t}$ and $\partial B_{0}=\partial B_{0}^{\phi} \cup \partial B_{0}^{q}$ such that $\partial B_{0}^{u} \cap \partial B_{0}^{t}=\emptyset$ and $\partial B_{0}^{\phi} \cap \partial B_{0}^{q}=\emptyset$ where the superscripts $u, t, \phi$, and $q$ denote the displacement, the traction, the electric potential, and the electric free charge density, respectively.

\subsection{Electrostatics equations}

According to the electrostatics theory, the electric displacement $\mathbb{D}$ is defined by the constitutive equation

$$
\mathbb{D}=\varepsilon_{0} \mathbb{E}+\mathbb{P},
$$

where $\mathbb{P}$ is the electric polarization density and $\varepsilon_{0}$ is the vacuum electric permittivity. The electric field $\mathbb{E}$ is conservative; therefore, it can be defined in terms of the electric potential $\phi$ :

$$
\mathbb{E}=-\nabla \phi
$$

The electric field $\mathbb{E}$ satisfies the following, which is called Faraday's law for electrostatics:

$$
\nabla \times \mathbb{E}=0
$$

where $\nabla \times \mathbb{E}$ is the curl operation. If there are no free currents or magnetic fields, the electric displacement $\mathbb{D}$ is expressed in the divergence:
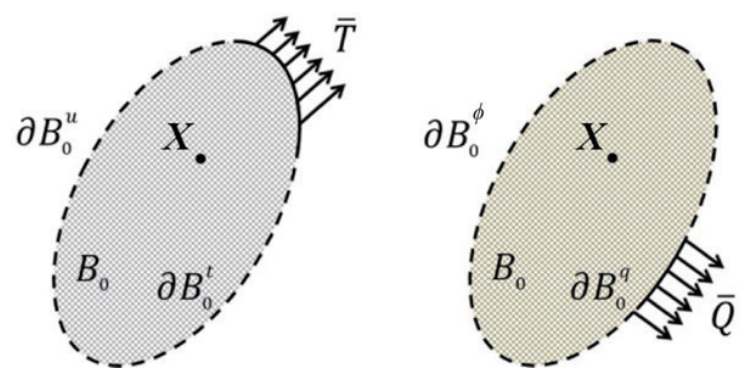

Fig. 2. (Color online) Body $B_{0}$ and its surface of each boundary defined as $\partial B_{0}=\partial B_{0}^{u} \cup \partial B_{0}^{t}$ and $\partial B_{0}=\partial B_{0}^{\phi} \cup \partial B_{0}^{q}$ such that $\partial B_{0}^{u} \cap \partial B_{0}^{t}=\emptyset$ and $\partial B_{0}^{\phi} \cap \partial B_{0}^{q}=\emptyset$ where the superscripts $u, t, \phi$, and $q$ denote the displacement, traction, electric potential, and electric free charge density, respectively. 


$$
\nabla \cdot \mathbb{D}=0
$$

where $\nabla \cdot \mathbb{D}$ is the divergence. At the boundary of the reference configuration, the electric displacement $\mathbb{D}$ must satisfy the continuity condition across the interfaces such that

$$
N \cdot \llbracket \mathbb{D} \rrbracket=\bar{q} \text { on } \partial B_{0}^{q}
$$

where $N$ is the unit outward normal vector to the reference configuration $B_{0}$ and is the free charge density on the surface $\partial B_{0}$. The notation $\llbracket \rrbracket$ is the jump operator, which is $\llbracket \mathbb{D} \rrbracket=\mathbb{D}^{\text {in }}-\mathbb{D}^{\text {out }}$, where the subscripts "in" and "out" denote the inside and outside of the boundary, respectively.

\subsection{Balance laws}

The balance laws serve as a fundamental set of equations for tackling boundary value problems or initial boundary problems with primary variables in continuum mechanics, which are composed of the balance of mass, linear momentum, angular momentum, and balance of energy. Firstly, the balance of mass is given in the integral form:

$$
\frac{D}{D t} \int_{B} \rho \mathrm{d} v=0
$$

where $\rho$ is the mass density. The local form of eq. (7) is expressed as

$$
\frac{\partial \rho}{\partial t}+\rho \nabla \cdot \boldsymbol{v}=0
$$

where $v$ is the velocity. The balance of linear momentum is

$$
\frac{D}{D t} \int_{B} \rho \boldsymbol{v} \mathrm{d} v=\int_{\partial B} \boldsymbol{t} \mathrm{d} a+\int_{B} \boldsymbol{b} \mathrm{d} v
$$

where $\boldsymbol{b}$ and $\boldsymbol{t}$ are the body force and surface traction, respectively. Considering elastostatics condition and applying the traction $\boldsymbol{t}=\boldsymbol{n} \boldsymbol{\sigma}$ to eq. (9) results in the following equilibrium equation:

$$
\nabla \cdot \boldsymbol{P}+\boldsymbol{b}=0
$$

where $\boldsymbol{P}$ is the first Piola-Kirchhoff stress. It is noted that equations expressed in either the current configuration or the reference configuration may generate the same result; however, in the present study, for brevity, most equations are given in the reference configuration. The boundary condition on the partial surface of the body to the equilibrium equation of eq. (10) is given in the form 


$$
\boldsymbol{P} \cdot \boldsymbol{N}=\overline{\boldsymbol{T}} \text { on } \partial B_{0}^{t},
$$

where $\overline{\boldsymbol{T}}$ denotes the surface traction at the boundary $\partial B_{0}^{t}$ shown in Fig. 2.

The balance of angular momentum is

$$
\frac{D}{D t} \int_{B} \rho \boldsymbol{x} \times \boldsymbol{v} \mathrm{d} v=\int_{\partial B} \boldsymbol{x} \times \boldsymbol{t} \mathrm{d} a+\int_{B} \boldsymbol{x} \times \boldsymbol{b} \mathrm{d} v
$$

After some complicated manipulation of eq. (12), the balance of the angular momentum equation turns out to be

$$
\operatorname{skew}(\boldsymbol{P}: \boldsymbol{F})=0 \text {. }
$$

When an electric field is activated, energy is stored in the material, generating a driving force and causing DEs to change in shape and size. The balance of energy states that the work done by stress as internal energy, the energy stored in the free space due to an electric field, and the polarization of the material are balanced by the external energy provided by external stimuli such as mechanical force and an electric field. The energy balance ${ }^{(25,26)}$ is given by

$$
\begin{aligned}
\frac{D}{D t} \int_{B}\left\{\frac{1}{2} \rho|\boldsymbol{v}|^{2}+e\right\} \mathrm{d} v= & \int_{\partial B} \boldsymbol{t} \cdot \boldsymbol{v} \mathrm{d} a+\int_{B} \boldsymbol{b} \cdot \boldsymbol{v} \mathrm{d} v \\
& -\int_{\partial B} \boldsymbol{q} \cdot \boldsymbol{n} \mathrm{d} a+\int_{B} \boldsymbol{r} \cdot \boldsymbol{v} \mathrm{d} v
\end{aligned}
$$

where $e$ is the internal energy and $\boldsymbol{r}$ is the energy supply due to an electric field. The energy balance equation is transformed into the local form

$$
\dot{e}=\boldsymbol{P}: \dot{\boldsymbol{F}}-\nabla \cdot \boldsymbol{Q}+\boldsymbol{R},
$$

where $\dot{e}$ is the material time derivative, $\boldsymbol{Q}$ is the heat flux and $\boldsymbol{R}$ is the energy supply in the reference configuration. The second law of thermodynamics is expressed in the form

$$
\int_{B} \gamma \mathrm{d} v=\int_{B} \dot{h} \mathrm{~d} v+\int_{\partial B} \frac{n \cdot \boldsymbol{q}}{\vartheta} \mathrm{d} a,
$$

where $\gamma$ is the entropy production, $h$ is the internal entropy and the second term on the right hand side of eq. (16) is the entropy flow. The local form of entropy inequality is obtained by applying the divergence theorem to eq. (16), which is 


$$
\Gamma=\dot{H}+\frac{\nabla \cdot \boldsymbol{Q}}{\vartheta}-\frac{1}{\vartheta^{2}} \boldsymbol{Q} \cdot \nabla \vartheta \geq 0
$$

where $\Gamma$ is the entropy production, which should be nonnegative, and $\dot{H}$ is the internal entropy in the reference configuration. Equation (17) being multiplied by the temperature $\vartheta$ gives two types of dissipation, which are the local contribution $\mathcal{D}_{\text {loc }}$ and the heat conduction contribution $\mathcal{D}_{\text {con }}$ as follows:

$$
\begin{aligned}
& \mathcal{D}_{\text {loc }}=\dot{H} \vartheta+\nabla \cdot \boldsymbol{Q} \geq 0, \\
& \mathcal{D}_{\text {con }}=-\frac{1}{\vartheta} \boldsymbol{Q} \cdot \nabla \vartheta \geq 0 .
\end{aligned}
$$

Arranging for the inequality equation with the heat conduction term $\nabla \cdot \boldsymbol{Q}$ eliminated in eqs. (15) and (18) gives

$$
\boldsymbol{P}: \dot{\boldsymbol{F}}+\boldsymbol{R}-\dot{H} \vartheta-\dot{e} \geq 0 .
$$

The free enthalpy $\Psi$ is defined in terms of the internal energy $e$, the internal entropy $H$, and the temperature $\theta$ as follows:

$$
\Psi=e-H \theta
$$

Taking the time derivative of eq. (21) gives

$$
\dot{\Psi}=\dot{e}-\dot{H} \theta-H \dot{\theta} .
$$

Substituting eq. (22) into eq. (20) and replacing $\boldsymbol{R}$ with $-\mathbb{D} \cdot \dot{\mathbb{E}}$ give the Clausius-Planck inequality

$$
\boldsymbol{P}: \dot{\boldsymbol{F}}-\mathbb{D} \cdot \dot{\mathbb{E}}-\dot{\Psi} \geq 0
$$

Since the free enthalpy $\Psi(\boldsymbol{F}, \mathbb{E})$ is a function of $\boldsymbol{F}$ and $\mathbb{E}$ according to eq. (23), the variation in time $\dot{\Psi}(\boldsymbol{F}, \mathbb{E})$ is given by

$$
\dot{\Psi}(\boldsymbol{F}, \mathbb{E})=\frac{\partial \Psi}{\partial \boldsymbol{F}}: \dot{\boldsymbol{F}}+\frac{\partial \Psi}{\partial \mathbb{E}} \cdot \dot{\mathbb{E}}
$$

Substitution of eq. (24) into eq. (23) gives the electromechanical constitutive equation: 


$$
\left(\boldsymbol{P}-\frac{\partial \Psi}{\partial \boldsymbol{F}}\right): \dot{\boldsymbol{F}}-\left(\mathbb{D}+\frac{\partial \Psi}{\partial \mathbb{E}}\right) \cdot \dot{\mathbb{E}} \geq 0
$$

or

$$
\boldsymbol{P}=\frac{\partial \Psi}{\partial \boldsymbol{F}}, \mathbb{D}=-\frac{\partial \Psi}{\partial \mathbb{E}}
$$

Yet another free energy $\widehat{\Psi}(\boldsymbol{F}, \mathbb{D})$, which is a function $\boldsymbol{F}$ and $\mathbb{D}$, is defined by the partial Legendre transformation

$$
\widehat{\Psi}(\boldsymbol{F}, \mathbb{D})=\Psi(\boldsymbol{F}, \mathbb{E})+\mathbb{D} \cdot \mathbb{E} .
$$

With the time derivative on eq. (27), the free energy $\widehat{\Psi}(\boldsymbol{F}, \mathbb{D})$ can be written in the form

$$
\dot{\Psi}(\boldsymbol{F}, \mathbb{D})=\dot{\Psi}(\boldsymbol{F}, \mathbb{E})+\dot{\mathbb{D}} \cdot \mathbb{E}+\mathbb{D} \cdot \dot{\mathbb{E}}
$$

The variation in time $\dot{\Psi}(\boldsymbol{F}, \mathbb{D})$ is given by

$$
\dot{\Psi}(\boldsymbol{F}, \mathbb{D})=\frac{\partial \widehat{\Psi}}{\partial \boldsymbol{F}}: \dot{\boldsymbol{F}}+\frac{\partial \widehat{\Psi}}{\partial \mathbb{D}} \cdot \dot{\mathbb{D}}
$$

In a similar fashion, substituting eq. (28) into eq. (23) with eq. (29) gives the inequality

$$
\left(\boldsymbol{P}-\frac{\partial \widehat{\Psi}}{\partial \boldsymbol{F}}\right): \dot{\boldsymbol{F}}-\left(\mathbb{E}-\frac{\partial \widehat{\Psi}}{\partial \mathbb{D}}\right) \cdot \dot{\mathbb{D}} \geq 0
$$

From eq. (30), meeting the inequality conditions, the constitutive equations are derived as

$$
\boldsymbol{P}=\frac{\partial \widehat{\Psi}}{\partial \boldsymbol{F}}, \mathbb{E}=\frac{\partial \widehat{\Psi}}{\partial \mathbb{D}}
$$

The available energy of a material can be best expressed through a free energy from classical thermodynamics. Upon the application of an electric field, energy is stored in the material in such a way that it is turned into a driving force causing different macroscopic and microscopic changes. It is noted that the work done by stress as internal energy, the energy stored in the free space due to an electric field, and the polarization all together are balanced by external energy. 
The principle of virtual work strictly requires that every term shown in a virtual equation must be of the same physical dimension such as the unit of work, which is called the "work conjugate". It is noted that $\boldsymbol{P}$ is the first Piola-Kirchhoff stress and the deformation gradient $\delta \boldsymbol{F}$ becomes work conjugates. Also, the electric field $\mathbb{E}$ and the electric displacement $\mathbb{D}$ are conjugated in work. It is important that primary variables should be work conjugates to be used for building a free energy function; otherwise, by failing the consistency in the principle of virtual work, one may get incorrect computation results. ${ }^{(28-33)}$

\section{Variational Formulation}

\subsection{Variational formulation}

With the equilibrium principles of thermodynamics, in order to determine the deformation subjected to an electric field, the constitutive equations are derived from the free energy function. Thereby, in order to set up a coupled multi-field analysis framework, the equations from electrodynamics and nonlinear continuum mechanics are to be brought into the same energy framework, which calls for the principle of virtual work. Upon applying the principle of virtual work, the equations can be transformed into integral equations, setting the displacement and the electric potential as independent variables. The integral equations derived through the application of the principle of virtual work need to be linearized with respect to the displacement and the electric potential to alleviate nonlinearity of the equations; otherwise, conventional linear numerical analysis cannot be performed properly to produce an approximate solution to the problems.

From the mechanical point of view, displacement, strain, stress, and deformation gradient may be chosen to be the primary variables of governing equations. As for the electric side, there are electric field, electric displacement, polarization, and electric potential. Depending on the solution framework, a different set of variables can be selected; however, it should be mentioned that the equation formulation must proceed only if those selected variables are work conjugates in the light of the principle of virtual work. Otherwise, numerical simulation may end up with ill-conditioned computations. Also, variables chosen should be measurable by instrument. The use of non-measurable entities as key variables does not guarantee the feasibility of numerical simulation results, which need to be compared with data from experiments. For example, interestingly, the nominal electric displacement and nominal electric field are work conjugates. In addition, the nominal stress and deformation gradient are also work conjugates.

Variational formulation requires the existence of a functional, which comprises all the governing equations and boundary and initial conditions together. The complexity of the procedure for solution to eqs. (3)-(6), (10), and (11) depends on what primary variables might be used in the computation, which determines a type of formulation with one variable or several variables. The development of a numerical procedure that is capable of finding the solution to the equations above along with the boundary conditions is a major goal in the present study. In this study, two different functionals- the primary Dirichlet functional and primal Hellinger-Reissner functional-are introduced and utilized up to the point where numerical frameworks are established. 


\subsection{Primal Dirichlet functional}

The primal Dirichlet-type functional ${ }^{(22)}$ has two independent variables, the deformation mapping $\boldsymbol{\eta}$ and the electric potential $\phi$. The functional may be considered as a maximization problem to which the solution variables are defined as

$$
(\phi, \boldsymbol{\eta})=\operatorname{Arg}\left\{\sup _{\phi} \inf _{\boldsymbol{\eta}} W(\phi, \boldsymbol{\eta})\right\}
$$

where the functional $W(\phi, \boldsymbol{\eta})$ is

$$
W(\phi, \boldsymbol{\eta})=\int_{B_{0}} \widehat{\Psi}(\boldsymbol{F}, \mathbb{E}) \mathrm{d} V-\int_{\partial B_{0}^{t}} \boldsymbol{\eta} \cdot \overline{\boldsymbol{T}} \mathrm{d} A+\int_{\partial B_{0}^{q}} \phi \bar{q} \mathrm{~d} A .
$$

Equation (33) is the primal Dirichlet-type functional composed of eqs. (3)-(5) and (10) with the corresponding boundary conditions of eqs. (6) and (11) for the surfaces where each boundary condition applies as shown in Fig. 2. Let the first variation of the functional of eq. (33) with respect to the deformation mapping $\boldsymbol{\eta}$ and the electric potential $\phi$ be $\delta W=0$, which is

$$
\delta W=\int_{B_{0}} \frac{\partial \widehat{\Psi}}{\partial \boldsymbol{F}}: \delta \boldsymbol{F} \mathrm{d} V+\int_{B_{0}} \frac{\partial \widehat{\Psi}}{\partial \mathbb{E}} \cdot \delta \mathbb{E} \mathrm{d} V-\int_{\partial B_{0}^{t}} \delta \boldsymbol{\eta} \cdot \overline{\boldsymbol{T}} \mathrm{d} A+\int_{\partial B_{0}^{q}} \delta \phi \bar{q} \mathrm{~d} A
$$

where $\delta \boldsymbol{\eta}$ is the virtual displacement and $\delta \phi$ is the virtual electric potential. The surface boundaries are $\partial B_{0}^{t}$ and $\partial B_{0}^{q}$, in which the virtual displacement and virtual electric potential are applied, respectively. Applying the Gauss theorem to eq. (34) gives

$$
\begin{aligned}
\delta W= & \int_{B_{0}}(\nabla \cdot \boldsymbol{P}) \cdot \delta \boldsymbol{\eta} \mathrm{d} V-\int_{B_{0}}(\nabla \cdot \mathbb{D}) \delta \phi \mathrm{d} V \\
& -\int_{\partial B_{0}^{t}}(\boldsymbol{P} \cdot \boldsymbol{N}-\overline{\boldsymbol{T}}) \cdot \delta \boldsymbol{\eta} \mathrm{d} A+\int_{\partial B_{0}^{q}}(\mathbb{D} \cdot \boldsymbol{N}-\bar{q}) \delta \phi \mathrm{d} A .
\end{aligned}
$$

Applying the stationary condition $\delta W=0$ to eq. (35) proves that the functional is correctly formulated because eqs. (3)-(5) and (10) along with boundary conditions of eqs. (6) and (11) are fully recovered from it. The next step is to perform the discretization and linearization to the functional of eq. (34) to obtain the solution to the problem.

\subsection{Primal Hellinger-Reissner functional}

As a second functional to be examined, the primal Hellinger-Reissner-type functional, (22) the primary variables being the deformation mapping $\boldsymbol{\eta}$, the electric displacement $\mathbb{D}$ and the electric potential $\phi$, is considered in order to obtain the solution of the following saddle point problem:

$$
(\phi, \mathbb{D}, \boldsymbol{\eta})=\operatorname{Arg}\left\{\sup _{\phi} \inf _{\mathbb{D}} \inf _{\boldsymbol{\eta}} W(\phi, \mathbb{D}, \boldsymbol{\eta})\right\}
$$


where the functional $W(\phi, \mathbb{D}, \boldsymbol{\eta})$ is

$$
W(\phi, \mathbb{D}, \boldsymbol{\eta})=\int_{B_{0}} \Psi(\boldsymbol{F}, \mathbb{D}) \mathrm{d} V+\int_{B_{0}} \mathbb{D} \cdot \nabla \phi \mathrm{d} V-\int_{\partial B_{0}^{t}} \boldsymbol{\eta} \cdot \overline{\boldsymbol{T}} \mathrm{d} A+\int_{\partial B_{0}^{q}} \phi \bar{q} \mathrm{~d} A
$$

Taking the first variation of the functional of eq. (37) with respect to the deformation mapping $\boldsymbol{\eta}$, the electric potential $\phi$ and the electric displacement $\mathbb{D}$ and letting $\delta W=0$,

$$
\begin{aligned}
\delta W= & \int_{B_{0}} \frac{\partial \Psi}{\partial \boldsymbol{F}}: \delta \boldsymbol{F} \mathrm{d} V+\int_{B_{0}} \frac{\partial \Psi}{\partial \mathbb{D}} \cdot \delta \mathbb{D} \mathrm{d} V+\int_{B_{0}} \delta \mathbb{D} \cdot \nabla \phi \mathrm{d} V \\
& +\int_{B_{0}} \nabla \delta \phi \cdot \mathbb{D} \mathrm{d} V-\int_{\partial B_{0}^{e}} \delta \boldsymbol{\eta} \cdot \overline{\boldsymbol{T}} \mathrm{d} A+\int_{\partial B_{0}^{e}} \delta \phi \bar{q} \mathrm{~d} A .
\end{aligned}
$$

Applying the Gauss theorem to eq. (38) gives

$$
\begin{aligned}
\delta W= & \int_{B_{0}}(\nabla \cdot \boldsymbol{P}) \cdot \delta \boldsymbol{\eta} \mathrm{d} V-\int_{B_{0}}(\nabla \cdot \mathbb{D}) \cdot \delta \phi \mathrm{d} V+\int_{B_{0}}(\mathbb{E}+\nabla \phi) \cdot \delta \mathbb{D} \mathrm{d} V \\
& -\int_{\partial B_{0}^{t}}(\boldsymbol{P} \cdot \boldsymbol{N}-\overline{\boldsymbol{T}}) \cdot \delta \boldsymbol{\eta} \mathrm{d} A+\int_{\partial B_{0}^{q}}(\mathbb{D} \cdot \boldsymbol{N}-\bar{q}) \delta \phi \mathrm{d} A .
\end{aligned}
$$

Equation (39) shows that the primal Hellinger-Reissner-type functional is built correctly because the system of equations, eqs. (3)-(5) and (10) along with the boundary conditions of eqs. (6) and (11) are retrieved.

\section{Numerical Framework}

\subsection{Discretization}

The weak form shown in eq. (34) or (38) needs to be discretized into finite elements. The domain $B_{0}$ is discretized into finite elements $B_{0}^{\mathrm{e}}$, that is, $B_{0}^{e}=\sum_{e=1}^{n_{\mathrm{el}}} B_{0}^{e}$, where $n_{\mathrm{el}}$ is the number of elements. The approximation of the geometry at the element level is expressed in terms of the nodal value $\boldsymbol{X}_{\alpha}$ at node $\alpha$ and the shape function $N_{\alpha}$ as

$$
\boldsymbol{X}=\sum_{\alpha=1}^{n_{\mathrm{en}}} N_{\alpha} \boldsymbol{X}_{\alpha}
$$

where $N_{\alpha}$ is the shape function of an element $e$ at node $\alpha$ in the local coordinate, and $n_{\mathrm{en}}$ is the number of nodes per element. In the present study, an isoparametric mapping is used as follows: 


$$
\boldsymbol{\eta}=\sum_{\alpha=1}^{n_{\mathrm{en}}} N_{\alpha} \boldsymbol{\eta}_{\alpha}, \phi=\sum_{\alpha=1}^{n_{\mathrm{en}}} N_{\alpha} \phi_{\alpha} .
$$

The variations of the variables are

$$
\delta \boldsymbol{\eta}=\sum_{\alpha=1}^{n_{\mathrm{en}}} N_{\alpha} \delta \boldsymbol{\eta}_{\alpha}, \delta \phi=\sum_{\alpha=1}^{n_{\mathrm{en}}} N_{\alpha} \delta \phi_{\alpha} .
$$

The nodal displacement and nodal electric potential of eqs. (40) and (41) are to be inserted into eq. (34) or (38) to generate discretized equations of the integral equations.

\subsection{Linearization of primal dirichlet functional}

The finite element residuals are obtained by substituting eqs. (40) and (41) into eq. (34) and rearranging it with variational variables:

$$
\begin{aligned}
& \boldsymbol{R}_{\boldsymbol{\eta}}^{\alpha}=\int_{B_{0}^{e}} \nabla N_{\alpha} \cdot \frac{\partial \Psi}{\partial \boldsymbol{F}} \mathrm{d} V-\int_{\partial B_{0}^{e}} N_{\alpha} \overline{\boldsymbol{T}} \mathrm{d} A \\
& \boldsymbol{R}_{\phi}^{\alpha}=-\int_{B_{0}^{e}} \frac{\partial \widehat{\Psi}}{\partial \mathbb{E}} \boldsymbol{B}_{\alpha} \mathrm{d} V+\int_{\partial B_{0}^{e}} N_{\alpha} \bar{q} \mathrm{~d} A .
\end{aligned}
$$

The residuals are used to set up an algebraic set of equations, to which the solution is found by the Newton-Raphson numerical method. Since DEs generate a large deformation under an electric field, the variational equation of eq. (34) becomes highly nonlinear. In order to solve nonlinear problems, eq. (34) should be linearized accordingly with respect to the deformation mapping $\boldsymbol{\eta}$ and the electric potential $\phi$; therefore, the Newton-Raphson method may be utilized. The linearized equation is $\delta W+$ $\Delta \delta W=0$, where $\Delta \delta W$ is the directional derivative with the increment of the deformation mapping $\Delta \boldsymbol{\eta}$ and the electric potential $\Delta \phi$. The linearized equation is given by $\Delta \delta W=$ $\Delta \delta W[\Delta \boldsymbol{\eta}]+\Delta \delta W[\Delta \phi]$.

Since the finite element residual $\boldsymbol{R}$ is nonlinear against the variable $\boldsymbol{d}$, the linearization with the Taylor series is executed as follows:

$$
\boldsymbol{R}^{k}+\frac{\partial \boldsymbol{R}^{k}}{\partial \boldsymbol{d}} \Delta \boldsymbol{d}^{k}=\boldsymbol{R}^{k+1}+\boldsymbol{K} \Delta \boldsymbol{d}^{k}=0
$$

where $k$ is the iteration index, the nodal variables $\{\boldsymbol{d}\}=\{\boldsymbol{\eta}, \phi\}$ and the residual $\{\boldsymbol{R}\}=\left\{\boldsymbol{R}_{\eta}, \boldsymbol{R}_{\phi}\right\}$.

$$
\boldsymbol{K}=\frac{\partial \boldsymbol{R}}{\partial \boldsymbol{d}} \quad, \boldsymbol{d}^{k+1}=\boldsymbol{d}^{k}+\Delta \boldsymbol{d}^{k+1}
$$


Equation (43) can be expressed in the matrix form

$$
[\boldsymbol{K}]\{\boldsymbol{d}\}=-\{\boldsymbol{R}\} .
$$

The element form of eq. (46) is expressed in the form

$$
\left[\begin{array}{ll}
\boldsymbol{K}^{\eta \boldsymbol{\eta}} & \boldsymbol{K}^{\boldsymbol{\phi} \phi} \\
\boldsymbol{K}^{\phi \boldsymbol{\eta}} & \boldsymbol{K}^{\phi \phi}
\end{array}\right]\left\{\begin{array}{c}
\Delta \boldsymbol{\eta} \\
\Delta \phi
\end{array}\right\}=-\left\{\begin{array}{l}
\boldsymbol{R}_{\boldsymbol{\eta}} \\
\boldsymbol{R}_{\phi}
\end{array}\right\},
$$

where the stiffness elements are

$$
\begin{gathered}
\boldsymbol{K}_{\alpha \beta}^{\eta \boldsymbol{\eta}}=\frac{\partial \boldsymbol{R}_{\boldsymbol{\eta}}^{\alpha}}{\partial \boldsymbol{\eta}_{\beta}}=\int_{B_{0}^{e}}\left(\boldsymbol{B}_{\alpha}: \frac{\partial^{2} \widehat{\Psi}}{\partial \boldsymbol{F} \partial \boldsymbol{F}}: \boldsymbol{B}_{\beta}\right) \mathrm{d} V, \\
\boldsymbol{K}_{\alpha \beta}^{\boldsymbol{\eta} \phi}=\frac{\partial \boldsymbol{R}_{\boldsymbol{\eta}}^{\alpha}}{\partial \phi_{\beta}}=-\int_{B_{0}^{e}}\left(\boldsymbol{B}_{\alpha}: \frac{\partial^{2} \widehat{\Psi}}{\partial \boldsymbol{F} \partial \mathbb{E}} \cdot \boldsymbol{B}_{\beta}\right) \mathrm{d} V, \\
\boldsymbol{K}_{\alpha \beta}^{\phi \boldsymbol{\eta}}=\frac{\partial \boldsymbol{R}_{\phi}^{\alpha}}{\partial \boldsymbol{\eta}_{\beta}}=-\int_{B_{0}^{e}}\left(\boldsymbol{B}_{\alpha} \cdot \frac{\partial^{2} \widehat{\Psi}}{\partial \mathbb{E} \partial \boldsymbol{F}}: \boldsymbol{B}_{\beta}\right) \mathrm{d} V, \\
\boldsymbol{K}_{\alpha \beta}^{\phi \phi}=\frac{\partial \boldsymbol{R}_{\phi}^{\alpha}}{\partial \phi_{\beta}}=\int_{B_{0}^{e}}\left(\boldsymbol{B}_{\alpha} \cdot \frac{\partial^{2} \widehat{\Psi}}{\partial \mathbb{E} \partial \mathbb{E}} \cdot \boldsymbol{B}_{\beta}\right) \mathrm{d} V,
\end{gathered}
$$

where $\boldsymbol{B}_{\alpha}=\nabla N_{\alpha}$. By solving eqs. (47) and (48) in a numerically iterative manner, the primary variables, the deformation mapping $\boldsymbol{\eta}$ and the electric potential $\phi$ are finally obtained.

\subsection{Linearization of primal Hellinger-Reissner functional}

In a similar fashion, the finite element residuals of eq. (38) are

$$
\begin{aligned}
\boldsymbol{R}_{\boldsymbol{\eta}}^{\alpha} & =\int_{B_{0}^{e}} \boldsymbol{B}_{\alpha} \cdot \frac{\partial \Psi}{\partial \boldsymbol{F}} \mathrm{d} V-\int_{\partial B_{0}^{e}} N_{\alpha} \overline{\boldsymbol{T}} \mathrm{d} A, \\
\boldsymbol{R}_{\mathbb{D}}^{\alpha} & =\int_{B_{0}^{e}} N_{\alpha} \frac{\partial \Psi}{\partial \mathbb{D}} \mathrm{d} V+\int_{\partial B_{0}^{e}} N_{\alpha} \boldsymbol{B}_{\alpha} \phi_{\alpha} \mathrm{d} V, \\
\boldsymbol{R}_{\phi}^{\alpha} & =\int_{B_{0}^{e}} \boldsymbol{B}_{\alpha} \cdot \mathbb{D}_{\alpha} N_{\alpha} \mathrm{d} V+\int_{\partial B_{0}^{e}} N_{\alpha} \bar{q} \mathrm{~d} A .
\end{aligned}
$$

The system of equation is given by 


$$
\left[\begin{array}{ccc}
\boldsymbol{K}_{\boldsymbol{\eta}} & \boldsymbol{K}^{\boldsymbol{D} \mathbb{D}} & 0 \\
\boldsymbol{K}^{\mathrm{D} \boldsymbol{\eta}} & \boldsymbol{K}^{\mathrm{DD}} & \boldsymbol{K}^{\mathrm{D} \phi} \\
0 & \boldsymbol{K}^{\phi \mathbb{D}} & 0
\end{array}\right]\left\{\begin{array}{c}
\Delta \boldsymbol{\eta} \\
\Delta \mathbb{D} \\
\Delta \phi
\end{array}\right\}=-\left\{\begin{array}{l}
\boldsymbol{R}_{\boldsymbol{\eta}} \\
\boldsymbol{R}_{\mathbb{D}} \\
\boldsymbol{R}_{\phi}
\end{array}\right\},
$$

where the stiffness elements are

$$
\begin{aligned}
& \boldsymbol{K}_{\alpha \beta}^{\boldsymbol{\eta} \boldsymbol{\eta}}=\frac{\partial \boldsymbol{R}_{\boldsymbol{\eta}}^{\alpha}}{\partial \boldsymbol{\eta}_{\beta}}=\int_{B_{0}^{e}} \boldsymbol{B}_{\alpha} \frac{\partial^{2} \Psi}{\partial \boldsymbol{F} \partial \boldsymbol{F}} \boldsymbol{B}_{\beta} \mathrm{d} V, \\
& \boldsymbol{K}_{\alpha \beta}^{\eta \mathbb{D}}=\frac{\partial \boldsymbol{R}_{\eta}^{\alpha}}{\partial \mathbb{D}_{\beta}}=\int_{B_{0}^{e}} \boldsymbol{B}_{\alpha} \frac{\partial^{2} \Psi}{\partial \boldsymbol{F} \partial \mathbb{D}} N_{\beta} \mathrm{d} V, \\
& \boldsymbol{K}_{\alpha \beta}^{\boldsymbol{\eta} \phi}=\frac{\partial \boldsymbol{R}_{\boldsymbol{\eta}}^{\alpha}}{\partial \phi_{\beta}}=0, \\
& \boldsymbol{K}_{\alpha \beta}^{\mathbb{D} \boldsymbol{\eta}}=\frac{\partial \boldsymbol{R}_{\mathbb{D}}^{\alpha}}{\partial \boldsymbol{\eta}_{\beta}}=\int_{B_{0}^{e}} N_{\alpha} \frac{\partial^{2} \widehat{\Psi}}{\partial \mathbb{D} \partial \boldsymbol{F}} \boldsymbol{B}_{\beta} \mathrm{d} V, \\
& \boldsymbol{K}_{\alpha \beta}^{\mathbb{D} \mathbb{D}}=\frac{\partial \boldsymbol{R}_{\mathbb{D}}^{\alpha}}{\partial \mathbb{D}_{\beta}}=\int_{B_{0}^{e}} N_{\alpha} \frac{\partial^{2} \Psi}{\partial \mathbb{D} \partial \mathbb{D}} N_{\beta} \mathrm{d} V, \\
& \boldsymbol{K}_{\alpha \beta}^{\mathbb{D} \phi}=\frac{\partial \boldsymbol{R}_{\mathbb{D}}^{\alpha}}{\partial \phi_{\beta}}=\int_{B_{0}^{e}} N_{\alpha} \boldsymbol{B}_{\beta} \mathrm{d} V, \\
& \boldsymbol{K}_{\alpha \beta}^{\phi \boldsymbol{\eta}}=\frac{\partial \boldsymbol{R}_{\phi}^{\alpha}}{\partial \boldsymbol{\eta}_{\beta}}=0, \\
& \boldsymbol{K}_{\alpha \beta}^{\phi \mathbb{D}}=\frac{\partial \boldsymbol{R}_{\phi}^{\alpha}}{\partial \mathbb{D}_{\beta}}=\int_{B_{0}^{e}} \boldsymbol{B}_{\alpha} N_{\beta} \mathrm{d} V, \\
& \boldsymbol{K}_{\alpha \beta}^{\phi \phi}=\frac{\partial \boldsymbol{R}_{\phi}^{\alpha}}{\partial \phi_{\beta}}=0 .
\end{aligned}
$$

By solving eqs. (50) and (51) in an iterative manner, the primary variables, the deformation mapping $\boldsymbol{\eta}$, the electric displacement $\mathbb{D}$ and the electric potential $\phi$ are determined.

\section{Discussion}

Theoretical frameworks that can deal with electro-mechanical coupling effects for DE have been developed for years, particularly associated with nonlinear continuum mechanics, providing a sound theoretical basis for multi-physics problems. 
With the help of nonlinear electro-continuum mechanics theory, numerical frameworks have been utilized to grasp the behavior of DE under multi-field external loads, the frameworks that employ the notion of the total stress and free energy. The total stress, which is the sum of the mechanically induced stress and electrically generated stress, is commonly used for the analysis. It is almost impossible to divide the total stress into the electrical stress and mechanical stress. The Maxwell stress is related to the force exerted by the electric field on charges in the material. A drawback of the total stress approach is that it is impossible to measure the physical quantities used in the analysis.

Alternatively, starting from the thermodynamic equilibrium theorem, the free energy function can be derived, not taking the total stress into consideration. Instead of nonmeasurable quantities such as Cauchy stress, electro-magnetic stress, and electric body force, measurable quantities can serve as independent variables. ${ }^{(29-33)}$ It is stated that the electric force or Maxwell stress cannot be defined correctly in dielectric media because it is no longer an operational concept. The use of measurable quantities for work conjugates such as nominal stress versus stretch and nominal electric field versus nominal electric displacement is proven right to produce physically correct solutions to the problems. It is important to acknowledge that a free energy function for which variational formulation may be obtained without employing the Maxwell stress, which turned out to be equivalent to those from others works. The free energy function consists of mechanical and electrical variables; therefore, a mathematical formulation for numerical analysis is set up via the principle of virtual work.

In order to generate a rigorous formulation in the principle of virtual work, one needs to keep equations of virtual work consistent in the light of work conjugates; otherwise it may result in an incorrect output. In this study, firstly, the fundamental kinematics and electrostatics eqs. (1) and (3)-(5), balance laws of electro-nonlinear-continuum mechanics of eqs. (7)-(18), and the free energy functions with primary variables are discussed in a consistent way.

Once a free energy function is chosen, applying the principle of virtual work to all the governing equations mentioned above gives the variational equations by considering them as minimization or saddle point problems with primary variables. The variational equations are thus discretized into the finite elements and linearized for the NewtonRaphson method to obtain the solution. In the present study, the whole procedure for setting up a numerical framework for the simulation of the behavior of dielectric elastomers is illustrated.

\section{Conclusion}

In the present study, the systematic detail in building a numerical framework for solving electro-mechanical problems such as the behavior of DE is illustrated clearly. The electro-mechanical problems on DEs are complicated so that it is quite confusing putting together all governing equations and physics laws from both fields into a unified framework. A transducer composed of dielectric elastomers and compliant electrodes is considered as an example. Conventional analysis employs the total stress that is divided 
into two different stresses: one is from mechanical loads and the other is due to an electric field.

However, it is impossible to measure the two different stresses separately that the recognition of the total stress may result in ambiguity. In this study, instead of using the total stress concept, a thermodynamics approach is adopted to establish the free energy function. With the free energy function, the principle of virtual work applies to build a weak form of the equations, with which the discretization and linearization are performed to be used in the finite element method. The advantage of the thermodynamic approach over other approaches, such as the principle of potential energy, is the sound theoretical background.

\section{References}

1 S. Klinkel: Int. J. Solids Struct. 43 (2006) 7197.

2 A. Ask, A. Menzel and M. Ristinmaa: Int. J. Non-Linear Mech. 47 (2012) 156.

3 A. Ask, A. Menzel and M. Ristinmaa: Mech. Mater. 50 (2012) 9.

4 C. Miehe and D. Rosato: Int. J. Eng. Sci. 49 (2011) 466.

5 D. Rosato and C. Miehe: Int. J. Eng. Sci. 74 (2014) 162.

6 C. Miehe and D. Rosato: Int. J. Eng. Sci. 49 (2011) 466.

7 C. Miehe, D. Zah and D. Rosato: Int. J. Numer. Meth. Engng. 91 (2012) 115.

8 C. Miehe and D. Zah: Comput. Methods Appl. Mech. Engrg. 267 (2013) 487.

9 M. Wissler and E. Mazza: Sens. Actuators A 138 (2007) 384.

10 A. Aczel: Procedia Eng. 48 (2012) 15.

11 D. L. Hennan, S. A. Chester and K. Bertoldi: J. Mech. Phys. Solids 61 (2013) 2047.

12 A. Dorfmann and R. W. Ogden: Q. J. Mech. Appl. Math. 57 (2004) 599.

13 D. K. Vu, P. Steinmann and G. Possart: Int. J. Numer. Mech. Engng. 70 (2007) 685.

14 D. K. Vu and P. Steinmann: Int. J. Fract. 147 (2007) 109.

15 D. K. Vu, P. Steinmann and G. Possart: Int. J. Numer. Mech. Engng. 70 (2007) 685.

16 D. K. Vu and P. Steinmann: Int. J. Solids Struct. 44 (2007) 7891.

17 D. K. Vu and P. Steinmann: Int. J. Fract. 147 (2007) 109.

18 R. Bustamante, A. Dorfmann and R. W. Ogden: Z. Angew. Math. Phys. 60 (2009) 154.

19 R. Bustamante, A. Dorfmann and R. W. Ogden: Int. J. Engng. Sci. 47 (2009) 1131.

20 R. Bustamante, A. Dorfmann and R. W. Ogden: Z. angew. Math. Phys. 60 (2009) 154.

21 D. J. Steigmann: Math. Mech. Solids 14 (2009) 390.

22 F. Vogel, R. Bustamante and P. Steinmann: Int. J. Non-linear Mech. 47 (2012) 341.

23 G. A. Maugin: Acta Mech. 35 (1980) 1.

24 G. A. Maugin: Int. J. Adv. Eng. Sci. Appl. Math. 1 (2009) 25.

25 R. M. McMeeking, C. M. Landis and S. M. A. Jimenez: Int. J. Non-Linear Mech. 42 (2007) 831.

26 R. M. McMeeking and C. M. Landis: J. App. Mech. 72 (2005) 581.

27 K. Y. Volkh: J. App. Mech. 79 (2012) 044507.

28 H. S. Park, Z. Suo, J. Zhou and P. A. Klein: Int. J. Solids Struct. 49 (2012) 2187.

29 Z. Suo, X. Zhao and W. H. Greene: J. Mech. Phys. Solids 56 (2008) 467.

30 Z. Suo: Acta Mech. Solida Sinica 23 (2010) 549.

31 Z. Suo, X. Zhao and W. H. Greene: J. Mech. Phys. Solids 56 (2008) 467.

32 Z. Suo: Acta Mech. Solida Sinica 23 (2010) 549.

33 R. E. Loge and Z. Suo: Acta Mater. 44 (1996) 3429. 